\title{
Intervention and Evocative Design Development (IEDD): Design experience through post-medical treatment and rehabilitation
}

\author{
Zakiyah Hasan ${ }^{1}$, Verly Veto Vermol 1, Rusmadiah Anwar 1, 2 \\ ${ }^{1}$ Faculty of Art \& Design, Universiti Teknologi MARA, 40450 Shah Alam Selangor, Malaysia \\ 2 National Design Centre, Faculty of Art \& Design, Universiti Teknologi MARA, 40450 Shah Alam Selangor, Malaysia \\ :zakiyahasan@uitm.edu.my, verly@uitm.edu.my, rusma935@uitm.edu.my, \\ Tel: +6013-3140107
}

\begin{abstract}
In medical and health care, product design and development play a key role in providing the optimal treatment and diagnosis with significant consideration of human factors, ergonomics, and practicality that meet the technical and clinical standards. Greater design attention through intervention and protocol analysis extended as a vital mechanism to evaluate the design efficacy throughout the treatment procedure is required. Design Intervention and Evocative Design Development (IEDD) is a materially innovative method explicitly oriented towards exploring the contemporary new understandings as a next-level towards the feasible solution.
\end{abstract}

Keywords: Design; Clinical; Human Factors; Procedures; Protocols

eISSN: 2398-4287 @ 2021. The Authors. Published for AMER ABRA CE-Bs by e-International Publishing House, Ltd., UK. This is an open access article under the CC BYNC-ND license (http://creativecommons.org/licenses/by-nc-nd/4.0). Peer-review under responsibility of AMER (Association of Malaysian Environment-Behaviour Researchers), ABRA (Association of Behavioural Researchers on Asians) and cE-Bs (Centre for Environment-Behaviour Studies), Faculty of Architecture, Planning \& Surveying, Universiti Teknologi MARA, Malaysia.

DOI: https://doi.org/10.21834/ebpj.v6iSI6.3046

\subsection{Introduction}

The demand for new and innovative, personalized medical devices, tools, or assistive aid is increasingly high each year. The advancement of design and manufacturing technology continuously adds more value to medical products and intervention in design features and functions. This emerging need for specific design products has led to rapid development and well-established benefits for patients with numerous medical treatments. As more inventions and innovations are explored in the development of medical devices, the design's effectiveness and practicality will undergo a series of continuous evaluations and assessments to improvise for betterment or to opt for a medical and clinical norm. Within the academic literature, a broad range of disciplines incorporate design evaluation and design intervention discussions and provide different perspectives of design process and development. Research on design intervention draws attention to explicitly introducing new findings and poses more open-ended research instruments to expose behavior, norms, and standards than as a resolution of the problem (or risen issues). It often challenges the conventional outcome of design evaluation and development of design processes, aesthetic or visual comfort, and performances. It articulates a new possibility to improve something or question discoveries. Utilizing basic design assessment and evaluation such as prototyping, remodeling, and reverse-engineering, design interventions are often more experimental, mischievous, and open-ended in setting up a frame for better explorative reliable insights. However, few scholars have researched how designers, expert users, and patients make sense of their experience with design betterment and explore ways in which constructions of newly developed design might influence their needs to improvise design criteria, ergonomics, and enhanced features and personalized values to a product. Such accounts rule out the possibility that the normative perspective of design evaluation and assessment, particularly those users and people involved within the application procedure or usage, might inhibit uncertainty from justifying design issues, performance, considerations, and engagement within the process. The concept of Personalized Health Care or Personalized Medicine was well documented (Lisa M. Meckley, Peter J. Neumann et al., 2010). It was first considered a

eISSN: 2398-4287 @ 2021. The Authors. Published for AMER ABRA cE-Bs by e-International Publishing House, Ltd., UK. This is an open access article under the CC BYNC-ND license (http://creativecommons.org/licenses/by-nc-nd/4.0/). Peer-review under responsibility of AMER (Association of Malaysian Environment-Behaviour Researchers), ABRA (Association of Behavioural Researchers on Asians) and cE-Bs (Centre for Environment-Behaviour Studies), Faculty of Architecture, Planning \& Surveying, Universiti Teknologi MARA, Malaysia.

DOI: https://doi.org/10.21834/ebpj.v6iSl6.3046 
novel strategy to tailor medical treatment to each patient's individual characteristics.

\subsection{The Need for Design Intervention}

Patient-specific diagnosis and treatments have recently been one of the most emerging research topics. Limitation of such innovation provided in hospitals, healthcare, and treatment centers will lead to patients' limitations in proper rehabilitation assistance. There is an emerging need to investigate design standards, methodologies, and technologies for robust, efficient, and effective product intervention guidelines for medical applications. This research explores the theoretical framework of design intervention in designing and evaluating the post-medical and rehabilitation treatment procedure.

Human-centered design is the central aspect of ergonomics; therefore, it needs to be considered when designing and developing specific medical products that can mainly be improvised during the design intervention phase (Vermol V.V., Anwar R., Hassan O.H. \& Abidin S.Z., 2017). Vermol et al. (2017) explained, researcher question (Why) by clarifying what the reality behind the designer's mind towards product cautious is and how users (patient) perceived them. Through design intervention, perhaps what designers think they know is what they do not know. (What) - only then, it determines the influence of experiencing themselves (designer) that they start to realize and reflect. (How) will discuss the appropriate way of understanding product factors through design intervention.

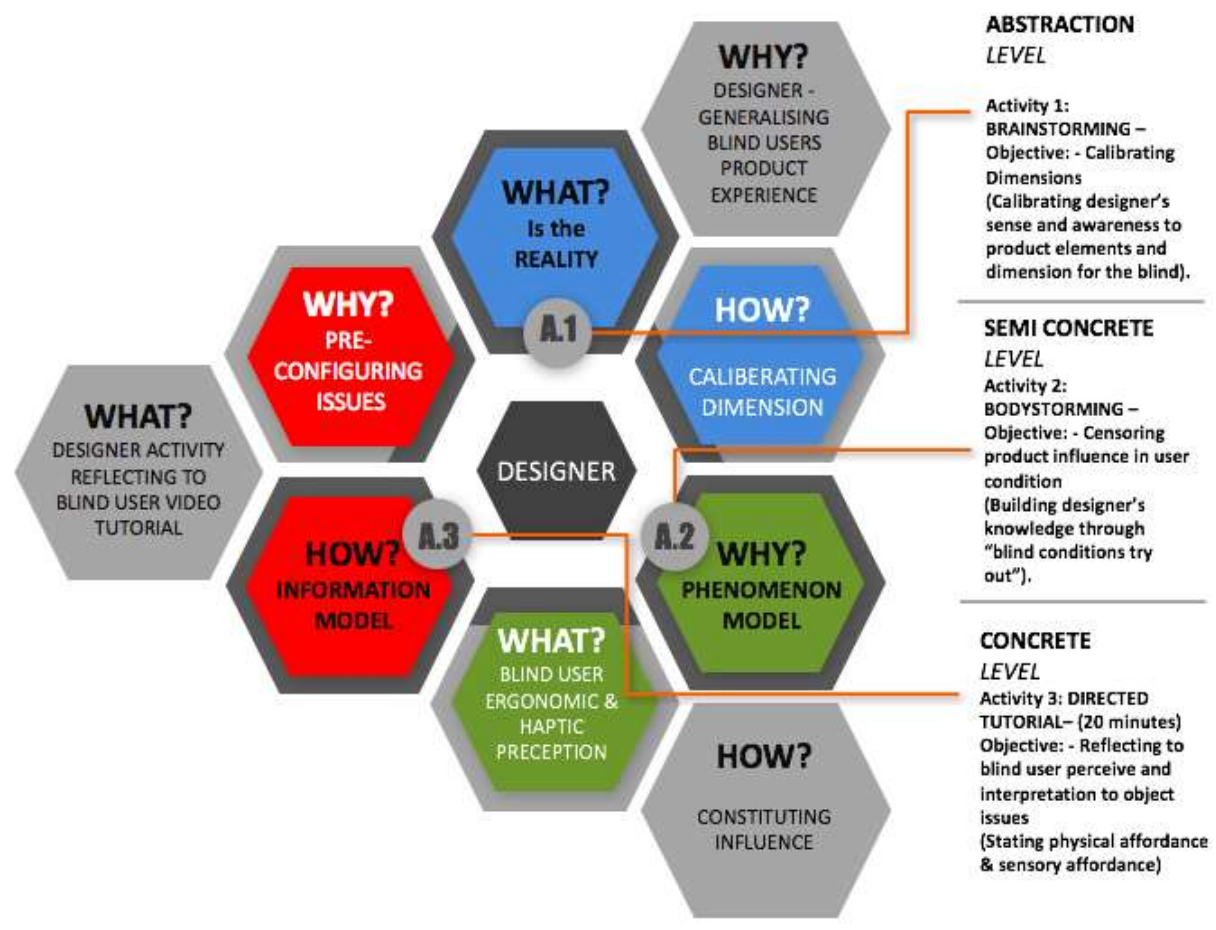

Figure 1: Conceptual model of designer activity (Vermol et al., 2017)

The common understanding of the design intervention model will allow more significant treatment planning, application areas, accuracy issues, and collaborative design within multi-disciplinary scope. The advantage of the design intervention mechanism will give a significant channel for future collaborative design and mass-customization needed to develop the optimal design and manufacturing systems of medical-related products.

\subsection{Literature Review}

\subsection{Factors contributing to Intervention \& Evocative Design Development (IEDD)}

Designing for users in a medical context, for example, patients or medical staff assistants, requires a deep understanding of their feelings, needs, and problems. The importance of gaining empathy through user research and various tools is discussed collectively, for example, by Sanders and Dandavate (1999) and Kouprie and Visser (2009). Jones (2013) elaborated on numerous problems that designers are nowadays facing when designing for healthcare. A user-centered design process focusing on empathy building seems to be the appropriate strategy for designing healthcare products (Wilke,2020). Due to the rapid evolvement of the design profession over the last decades towards developing a methodical, evidence-based, and holistic process, it is critical for the designer with a user-centered design approach to conduct user research. Although this might be common among designers, it is not always a shared perception of other professions, which leads to a disregard of design research (Noël and Frascara, 2016) and restricts designers to the concept-phase of the design process. 


\subsection{Medical Product Design, Methods, and Applications}

Specialized healthcare and medical products in particular aim to provide the optimal diagnosis and treatment with the use of the right medicine, tools, and devices to the right patient at the right time in order to meet well technical and clinical requirements as well as individual characteristics of each patient (Hieu Le and Okereke et al., 2011). The typical medical product and applications widely been personalized include i) Biomodels, ii) Surgical tools and devices, iii) Ergonomics, orthotics, and prostheses, v) Implants for bone reconstruction, vi) Scaffolds for tissue engineering; and (vii) Patient-specific contact lens. Most of the time, designers need to collaborate with MD or specialists for treatment or therapy. Design is carefully evaluated and optimized before it is transferred to manufacturing processes for production. In the standard practice of current case studies related to Product Design Process (PDP) in the medical context, these data will be assessed, and input will be adapted for design process and reasoning: i) problem interest, ii) patient-specific preference, iii) patient's anatomy, iv) pre-operative planning and risk measure, v) Procedure rehearsal and optimization, vi) Pre-fabricate solution, custom-made assistive aid, and module integration, vii) evaluation and assessment of effectiveness. More and more high-value-added personalized products are being developed to meet patient-specific requirements to improve the quality of diagnosis and treatment for patients. However, there are challenges that need to be overcome in order to make it possible for the wide applications in hospitals in both developed and developing countries (Hieu Le and Okereke, et al, 2011).

\subsection{Rehabilitation}

Physical medicine and rehabilitation cover a variety of clinical settings and patient populations. It is also known as a physiatrist (Lee, 2011). that aims to enhance and restore functional ability and quality of life to people with physical impairments or disabilities. Physical medicine also significantly refers to post-medical treatment with some of the procedures that will take place in a non-clinical environment. This stage of post-medical treatment assists a person in functioning optimally within the limitations placed upon the patients by a disabling impairment or disease process for which there is no known cure. Comprehensive rehabilitation is given by specialists in this field, who perform as facilitators, team leaders, and medical experts for rehabilitation (Levack et al, 2015).

\subsection{Occupational Therapy in Medical Treatment}

Occupational therapy (OT) refers to a branch of health care that deals with all ages of people with cognitive, physical, sensory problems and helps people with barriers that affect a person's physical, emotional, and social needs (Himabindhu, 2020). This post-medical treatment will involve specific routine and rehabilitation procedure activities, exercises, and other therapies daily until the patient completes the standard of designated treatment level, improvement stage, or recovery stage. The need to develop fine motor skills in OT requires special equipment to help build their independence. These include wheelchairs, splints, bathing equipment, assistive devices, and communication aids. Himabindhu (2020) outlined that there is a difference between occupational therapy and physical therapy. Occupational therapy focuses on improving the client's ability to perform activities of daily activities (ADL). Physical therapy focuses on improving the client's ability to perform a movement of the human body.

Given a specific spectrum, OT involves advanced measures to cater to the patient's ability beyond the physical requirement. Occupational therapy helps with:

- Fine motor skills (small-muscle movements made with the hands, fingers, and toes, such as grasping)

- Visual-perceptual skills

- Cognitive (thinking) skills

- Sensory-processing problems

Based on the preliminary case studies, specialized exercises and treatment modules are designed to support the functions of targeted therapy procedure, rehabilitation requirement, and need. Although occupational therapists assist people by analyzing the patient and finding out the best suited for psychological, emotional, physical, environmental, and needs, there are interventions needed to continuously improve the procedure and product design system related to the scope of exercise or modules. Due to the need for post-treatment care and occupational therapy among patients with disabilities, a comprehensive design framework is vital to continuously help designers, medical experts, and patients access more excellent design experiences. Increasing numbers of these groups of patients mismatch the capability and affordance to access good rehabilitation treatment due to the lack of assistive devices for specialized therapy procedures.

\subsection{Clinical Procedure}

A clinical procedure can be defined as any health practitioner practice involving a combination of special skills or abilities that may require drugs, devices, or both. However, clinical procedures are not essentially dependent on new health care products but the provider's technique performing the procedure. For example, the development of certain surgical procedures (although they may involve the use of scalpels, clamps, and drugs) or psychotherapy (Kievit, W et al. 2017). According to Gelijns (2002), the development of incremental innovations in clinical procedures usually occurs in a much more decentralized fashion, involving numerous physicians refining and modifying an existing procedure in everyday clinical practice. In contrast to medical device innovation, which requires the bridging of "collaborative cultures" (that of designers, engineers, and clinical researchers), the distinction between "developers" and "evaluators/users" may be very fine or even non-existent in the development of clinical procedures. The design process, product, and usage evaluation often been inducted by the technical requirement and lack of human-centered considerations. To date, the potential safety, efficacy, and effectiveness of many procedures have not been evaluated systematically during their development. (Kievit, W et al. 2017). According to Wennberg, many procedures have not received careful feasibility studies during their initial application in humans. However, they have 
been introduced based on investigations involving patient historical controls or more anecdotal evidence during the design-experience evaluation.

Figure 2: Research design Sequential Exploratory Design in IEDD

SEQUENTIAL EXPLORATORY DESIGN

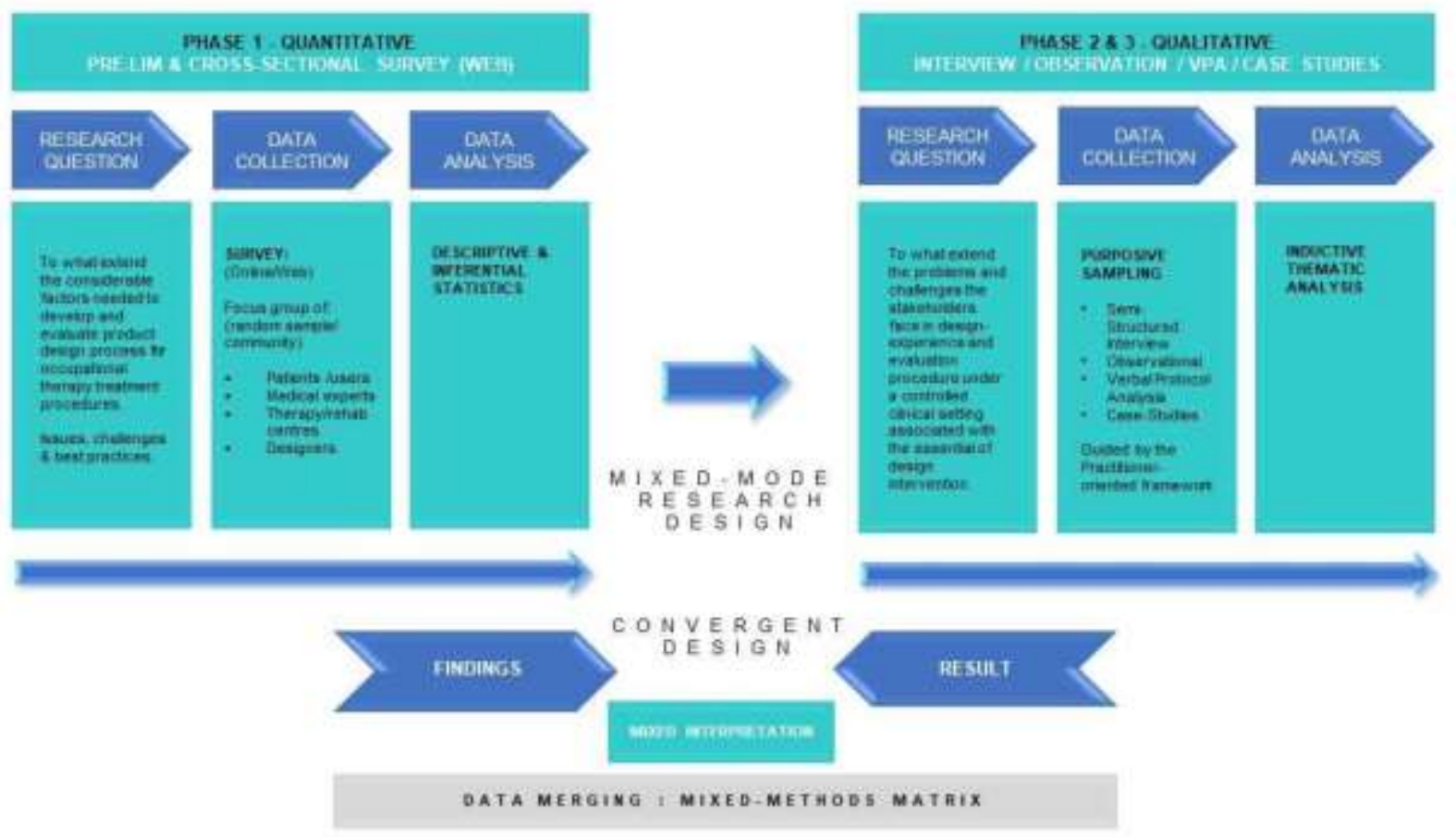

\subsection{Research Design}

Exploration of these objectives requires an approach that describes relationships between variables and the experiences of users and engagement of other participants during the design evaluation and assessment. Therefore, the research aims to suit a mixed-method approach. The research consists of two components: quantitative and qualitative methods to examine i) the product usage responses (among user experts and patients), ii) the design evaluation practice and understanding (among designers and expert users), and iii) to pursue deep insights from three major groups of i) patients, ii) expert users - doctors, medical assistance, nurses, therapist, or caregivers, and iii) designers about the design aspects, human factors, and product feasibility. The related perspectives employed in this study permits a quantitative investigation in which design intervention intersects with the aspects of human factors, environment, behavior, norms or routine, and services. Complimenting this aspect is the use of i) in-depth interviews to ask on user's experiences of product design, usage, and accessing the services for post-treatment, with further investigation using ii) verbal protocol analysis VPA to ask on designer's input on design evaluation and possible design intervention on selected product/prototype, and iii) selected of four case studies sample to engage with the actual process and procedure of related design project for post-medical product design. This approach reveals how results pertaining to the evaluation of a product design are insufficient to justify the need for proper design improvement and, more importantly, affect the designer's constructed notion of design intervention. To achieve this research aim, a further systematic method of a socially significant research question and observation will be focusing on which concerning the contribution to the literature of design process in post-medical treatment and rehabilitation studies. The quest begins by introducing the researcher's research models in staging the design activity of this study.

As for research outcome, it is crucial to decide an approach that fits the goal, ambitions, and resources to determine the necessary activities without compromising the study's allocated resources and limitations. Explanations of the design process, evaluation, and guidelines in clinical procedure will be mapped down to visualize a more apparent flow and steps taken for this research and manage the expectations and preparation of this research. This research approach will also consider the data collection procedures, explaining the type of research that was carried out. Finally, the method of data collection and analysis will also be rationalized. The researcher thought that neither quantitative or qualitative approach by itself is adequate to grasp the research problem fully; hence, as supported by the statements from Creswell (2009), a mixed-method design is preferred.

As this research utilized the mixed-method design approach, both quantitative and qualitative data will be collected sequentially. This study starts with a broad survey to generalize findings to a population and stakeholders before focusing on qualitative, semi-structured interviews to collect detailed opinions from the informers. In designing the study using a mixed-method design, four factors contribute to the procedures of this study - timing, weight, mixing, and theorizing. The researcher has chosen the Sequential Explanatory Design to easily communicate the procedures for this mixed-method study, as adapted from Morse (1991), Tashakkori and Teddlie (1998), Creswell 
and Plano Clark (2007), and summarized by Creswell et al. (2003). Therefore, Sequential Explanatory Design strategy as shown (see Methodology. Fig.4.0: Sequential Explanatory Design strategy in IEDD will be the starting point of the activity of preliminary data collection and analysis through quantitative approach during the first phase of the research and followed by the collection and analysis of qualitative data, which was build based on the results from the first quantitative study. Ethics and code of research conduct will be applied prior to the research study initiation. This research will be conducted within these four empirical phases:

\section{- Phase 1: THEORETICAL RESEARCH ACTIVITY}

Preparation \& Preliminary (Literature Review)

Identifying Theories, issues, challenges, and best practices

\section{- Phase 2: INQUIRY RESEARCH ACTIVITY}

Primary Data Collection (Exploratory Research)

Exploring and synthesizing theories, issues, challenges \& best practices

- Phase 3: PARTICIPATORY RESEARCH ACTIVITY

Research and evaluation in the design \& execution process

Ensuring methods and findings reflects the objectives \& perspectives of subjects

- Phase 4: DATA ANALYSIS, REPORTING, AND REFLECTION

Discussion on data findings \& results

Establishing conventions and framework

\subsection{Findings}

The experiments, results, and reviews give some additional inquiries concerning the interaction between user, designer, and expert's perception going across on two (2) subject modalities unit mediated through the context of human-centered and design performances. The consequences of both reviews appear to have recommended that inside methodology, engagement and experience may improve judgment, more in accordance with the work of Saema \& Claus (2015) that fundamental to understand and support design practice. Moreover, the importance of understanding the standard practice is towards design reasoning, where this process involves cognitive activity that dictates how users or patients respond to situations in every aspect of their lives. Design activity relies on the reasoning processes of designers. Therefore, understanding the role of reasoning and experiences in design is critical to understanding how design occurs in a medical context. The experiments, results, and reviews give some additional inquiries concerning the interaction between user, designer, and expert's perception going across on two (2) subject modalities unit mediated through the context of human-centered and design performances. The consequences of both reviews appear to have recommended that inside methodology, engagement and experience may improve judgment, more in accordance with the work of Saema \& Claus (2015) that fundamental to understand and support design practice. Moreover, the importance of understanding the standard practice is towards design reasoning where this process involves cognitive activity that dictates how users or patients respond to situations in every aspect of their lives. Design activity relies on the reasoning processes of designers. Therefore, understanding the role of reasoning and experiences in design is critical to understanding how design occurs in a medical context.

\subsection{Discussion}

The program's ability to handle a large set of variables allows mass data to be collected and statistically analyzed. The data will be analyzed for the qualitative collection of data by identifying the common patterns within the responses. The patterns will then be analyzed in a way that is in line with the research objectives. Observation will be conducted and sequentially analyzed onsite. Data will be collected through notes, photos, and video recording. Typically, the weightage of collected feedback data will be analyzed through the quantitative approach, determining the variables for the qualitative phase. The implication from the research of this research introduces the fundamental nature of user and experience evaluation in the design process and its critical knowledge that will result from the protocol experiments conducted to influence the direction of product design development towards user-designers cognitive in collaborative thinking.

\subsection{Conclusion \& Recommendations}

Innovation and new product development in the medical device industry have largely been technology-driven in the last decades but always to solve clinical needs. Advancements have often been rapid and significant. Today's medical-related products have reached high standards; consequently, the state of art treatment generally supports an acceptable return to daily activities and quality of life. Nonetheless, today's product development occurs in a complex healthcare environment, with multiple stakeholders involved. Each of these stakeholders can have different and sometimes conflicting requirements and expectations. While technological innovation to solve current clinical needs may still be the driving force behind new product development, it is not sufficient to reach the clinic in many cases. Consequently, this study and research findings will benefit significantly to broader areas of applications such as Medical Design Research \& Development, Medical Product Business, Manufacturing \& Supplies, Healthcare, Rehabilitation Centre, and Special-Treatment 
Centers/Clinics, Design, Health Science \& Clinical Education, Collaborative Research and Design Project for Medical Device, Ergonomics, Human-Factors, and User-centered Design, Medical Design Technology (e-health) and Data-driven Patient-Preference.

\section{Acknowledgements}

The authors would like to acknowledge the Research Management Center (RMC), Universiti Teknologi MARA, for funding under the grants of the 600-IRMI/FRGS 5/3 (463/2019) for this publication.

\section{References}

AC Gelijns, SO Their (2002). Medical innovation and institutional interdependence: rethinking university-industry connections. Jama 287 (1), $72-77$.

Abbas Tashakkori, Charles Teddlie, Charles B. Teddlie (1998). Mixed methodology: Combining qualitative and quantitative approaches. SAGE. Applied Social Research Methods Series. Vol 46. ISBN: 9780761900719.

Annetine C. Gelijns (1989).Technological Innovation: Comparing Development of Drugs, Devices, and Procedures in Medicine; Institute of Medicine (US) Committee on Technological Innovation in Medicine. Washington (DC): National Academies Press (US); 1989.

Bates S. (2010) "Progress towards personalized medicine" Drug Discov Today. 2010 Feb;15(3-4):115-20. https://doi.org/10.1016/j.drudis.2009.11.001.

C.H Le, M.I Okereke, V.H Nguyen, V.D Dao, N Zlatov, V.T Nguyen, T.H Le. Personalised Medical Product Development: Methods, Challenges and Opportunities. (2011). In proceedings of the International Conference on Innovations, Recent Trends And Challenges In Mechatronics, Mechanical Engineering And New High-Tech Products Development - MECAHITECH'1.

Ciccone, N.W., Patou, F. and Maier, A.M. (2019), "Designing for Better Healthcare: A Systemic Approach Utilising Behavioural Theory, Technology and an Understanding of Healthcare Delivery Systems", International Conference on Engineering Design, Vol. 1 No. 1, pp. 937-946.

Creswell, J. W., \& Plano Clark, V. L. (2007). Designing and conducting mixed methods research. Thousand Oaks, CA: Sage.

Creswell, J. W., Plano Clark, V. L., Gutmann, M. L., \& Hanson, W. E. (2003). An expanded typology for classifying mixed methods research into designs. Advanced mixed methods research designs. In A. Tashakkori \& C. Teddlie (Eds.), Handbook of mixed methods in social and behavioral research (pp. 209-240). Thousand Oaks, CA: Sage.

Daan J.A. Crommelin, Storm G., and Luijten P. (2011) "'Personalized medicine' through 'personalized medicines': Time to integrate advanced, non-invasive imaging approaches and smart drug delivery systems". International Journal of Pharmaceutics. 2011; 30;415(1-2):5-8.

E. Van Overbeeke, R. Janssens, C. Whichello, K. Schölin Bywall, et al. (2019). "Design, Conduct, and Use of Patient Preference Studies in the Medical Product Life Cycle: A Multi-Method Study". Frontiers in Pharmacology. Vol 10. Article 1395. DOI: 10.3389/fphar.2019.01395.

Fokkinga, S. F., Desmet, P. M. A., \& Hekkert, P. (2020). Impact-centered design: Introducing an integrated framework of the psychological and behavioral effects of design. International Journal of Design, 14(3), 97-116.

Godbold, R., Lees, A. and Reay, S. (2019), "Ethical Challenges for Student Design Projects in Health Care Settings in New Zealand", International Journal of Art \& Design Education, Vol. 38 No. 1, pp. 182-192.

Goodrich, J. (2018), "Why experience-based co-design improves the patient experience", The Journal of Health Design, Vol. 3, No. 1 (2018)

Himabindhu G (2020). Occupational Therapy. International Journal of Neurorehabilitation 7(5) DOI: 10.37421/ijn.2020.7.373.

H. Wilke , P. Badke-Schaub and K. Thoring (2020). The Healthcare Design DilemmA: Perils of a Technology-Driven Design Process for Medical Products. DOI: 10.1017/dsd.2020.133. DESIGN Conference. Volume: 1 (2217-2226).

John W. Creswell (2009). Editorial: Mapping the Field of Mixed Methods Research. Journal if Mixed Methods Research. https://doi.org/10.1177/1558689808330883.

John W. Creswell \& Wanqing Zhang (2009). The application of mixed methods designs to trauma research. Journal of Traumatic Stress. Volume22, Issue6 December 2009. Pages 612-621. https://doi.org/10.1002/jts.20479.

Juhyeok Park, Eungdo Kim, Kwangsoo Shin, (2019). Developing an Evaluation Framework for Selecting Optimal Medical Devices. Journal of Open Innovation: Technology, Market, and Complexity.

Kievit, W., Tummers, M., Van Hoorn, R., Booth, A., Mozygemba, K., Refolo, P., et al. (2017). Taking patient heterogeneity and preferences into account in health technology assessments. Int. J. Technol. Assess. In Health Care 33 (5), 562-569. DOI: 10.1017/S0266462317000885.

Kouprie M. Froukje and Visser S. (2009) A framework for empathy in design: Stepping into and out of the user's life. October 2009.Journal of Engineering Design 20(5):437448. DOI: $10.1080 / 09544820902875033$.

Lee, Peter K. W. (2011). "Defining Physiatry and Future Scope of Rehabilitation Medicine". Annals of Rehabilitation Medicine. 35 (4): 445-449. doi:10.5535/arm.2011.35.4.445. PMC 3309231. PMID 22506158. 
Levack, William M. M.; Weatherall, Mark; Hay-Smith, E. Jean C.; Dean, Sarah G.; McPherson, Kathryn; Siegert, Richard J. (2015). "Goal setting and strategies to enhance goal pursuit for adults with acquired disability participating in rehabilitation". The Cochrane Database of Systematic Reviews (7): CD009727. doi:10.1002/14651858.CD009727.pub2. hdl:10292/9134. ISSN 1469-493X. PMID 26189709.

Lis Meckley, Lisa M. \& Neumann, Peter J. (2010) "Personalized medicine: Factors influencing reimbursement," Health Policy, Elsevier, vol. 94(2), pages 91-100, February.

McCarthy, G. M., Rodríguez Ramírez, E. R., \& Robinson, B. J., (2017). Design Experiments. Identifying areas for intervention and designing medical technology for adolescents and young adults with type 1 diabetes. The Design Journal: An International Journal for All Aspects of Design. DOI: 10.1080/14606925.2017.1352723.

Morse, Janice M. RN (1991). Approaches to Qualitative-Quantitative Methodological Triangulation. Nursing Research: March 1991 - Volume 40 - Issue 2 - p $120-123$.

Noël G., Frascara J., \& Wong C. (2016). Designing bowel preparation patient instructions to improve colon cancer detection: Evidence-based design criteria for patients' documents. December 2019. Information Design Journal 25(1). DOI: 10.1075/idj.25.1.09noe.

Peter H. Jones (2013) Design for Care: Innovating Healthcare Experience. January 2013. Publisher: Rosenfeld Media. ISBN: 1933820233.

Sanders, E.B.-N \& Uday Dandavate (1999). Proceedings of the First International Conference on Design and Emotion, C.J. Overbeeke and P. Hekkert (Eds.), TU Delft, 1999.

Vermol V.V., Abidin S.Z., Anwar R., Hassan O.H. (2018) Blind User Experience Audit: Revealing Underlying Invisible Factors in Design Experience. In: Anwar R., Mahamood M., Md. Zain D., Abd Aziz M., Hassan O., Abidin S. (eds) Proceedings of the Art and Design International Conference (AnDIC 2016). Springer, Singapore. https://doi.org/10.1007/978-981-13-0487-3_55

Vermol V.V., Anwar R., Hassan O.H. \& Abidin S.Z. (2017) Designer Activity Experience: Blind UserDesigner Activity Model In Knowing Product Influence Through Blind User Perspective. Adv. Sci. Lett. 23, Pg. 10815-10821 (2017)

Vermol V.V., Abidin S.Z., Anwar R., Hassan O.H. (2018) Blind User Experience Audit: Revealing Underlying Invisible Factors in Design Experience. In: Anwar R., Mahamood M., Md. Zain D., Abd Aziz M., Hassan O., Abidin S. (eds) Proceedings of the Art and Design International Conference (AnDIC 2016). Springer, Singapore. https://doi.org/10.1007/978-981-13-0487-3_55 